\title{
Résilience Communautaire Des Riverains Autochtones Batwa Dans Le Contexte Du Conflit Au Parc National De Kahuzi Biega, Est De La Rd. Congo
}

\author{
Mukumba Isumbisho Pacifique, \\ Doctorant à l'École Doctorale de l'Université du Burundi, Chercheur au \\ Centre de Recherche pour la Promotion Rurale (CERPRU) de l'Institut \\ Supérieur de Développement Rural de Bukavu (ISDR-Bukavu), \\ République Démocratique du Congo
}

\section{Manirakiza René,}

Professeur à l'Université du Burundi

URL:http://dx.doi.org/10.19044/esj.2020.v16n29p83

\section{Résumé}

Introduction : Les délocalisations des peuples autochtones de leurs terres ancestrales en faveur de la création des réserves naturelles ou aires protégées datent de quelques siècles et se sont effectuées dans plusieurs pays. Elles ont suscité des conflits entre les peuples autochtones et les gestionnaires des aires protégées et produit un état de choc chez les autochtones riverains Batwa dans le cas de la RDC. L'étude vise à identifier les initiatives de résiliences communautaires développées par les riverains autochtones Batwa dans le contexte de conflits, dans la partie haute altitude du Parc National de Kahuzi Biega à l'Est de la RDC. Méthodologie : Les données utilisées dans cette étude ont été collectée au moyen des discussions de groupes menées, accompagnées des interviews approfondies avec certaines personnes ressources et les leaders autochtones Batwa. Résultat : Les résultats montrent que vingt-trois (23) initiatives résilientes communautaires ont été identifiées dont cinq (5) initiatives endogènes et dix-huit (18) exogènes. Les initiatives endogènes de résilience ont eu plus d'impact significatif dans le mode de vie des riverains autochtones Batwa que les initiatives exogènes de résilience. Conclusion: Les initiatives de résiliences communautaires auxquelles s'adonnent les riverains autochtones Batwa dans le milieu d'accueil, sont juste à classer dans leur capacité d'adaptation et non de transformation dans l'hinterland du Parc National de Kahuzi Biega car cela nécessite les mécanismes de gouvernance, les politiques / réglementations, l'infrastructure, les réseaux communautaires et les mécanismes formels de protection sociale qui font partie du système plus vaste dans lequel les communautés doivent s'intégrer. 
Mots clés : Résilience communautaire, initiative exogène, initiative endogène, Kahuzi Biega, autochtone Batwa, conflit

\title{
Community Resilience of Batwa Indigenous Peoples in the Context of Conflict at Kahuzi Biega National Park, East of Democratic Repubic of Congo
}

\author{
Mukumba Isumbisho Pacifique, \\ Doctorant à l'École Doctorale de l'Université du Burundi, Chercheur au \\ Centre de Recherche pour la Promotion Rurale (CERPRU) de l'Institut \\ Supérieur de Développement Rural de Bukavu (ISDR-Bukavu), \\ République Démocratique du Congo \\ Manirakiza René, \\ Professeur à l'Université du Burundi
}

\begin{abstract}
Introduction: The relocation of indigenous peoples from their ancestral lands in favor of the creation of reserves or protected areas dates back a few centuries and has taken place in several countries. This has led to conflict between indigenous peoples and protected area managers. These evictions have produced a state of shock among the indigenous Batwa riverside dwellers. The study aims to identify community resilience initiatives developed by indigenous Batwa riverine dwellers in the context of conflict in the high altitude part of Kahuzi Biega National Park in eastern DRC. Methodology: The data used in this study was collected through focus groups discussion, accompanied by in-depth interviews with resource persons and Batwa indigenous leaders. Result: The results show that twenty three (23) community resilience initiatives were identified, including five (5) endogenous community resilience initiatives and eighteen (18) exogenous community resilience initiatives. The endogenous resilience initiatives had a more significant impact on the lifestyle of Batwa indigenous residents than the exogenous resilience initiatives. Conclusion: The community resilience initiatives that Batwa Indigenous neighbors are engaged in the host environment are aptly categorized as adaptive rather than transformative in the Kahuzi Biega National Park hinterland as this requires governance mechanisms, policies/regulations, infrastructure, community networks and formal social protection mechanisms that are part of the wider system into
\end{abstract}


which communities need to integrate.

Keywords: Community resilience, exogenous initiative, endogenous initiative, Kahuzi Biega, indigenous Batwa, conflict

\section{Introduction}

La résilience des communautés est une notion qui cherche à expliquer leur capacité à réagir à un choc. Cette notion qui peut être examinée comme un sous-champ de la résilience sociale (Matarrita-Cascante et al., 2017), est l'un de principe de la performance de bonne gouvernance pour les aires protégées (Borrini-Feyerabend et al., 2014). La résilience communautaire des autochtones Batwa (Pygmée) riverains vivant dans l'hinterland du Parc National de Kahuzi Biega (PNKB) dans leurs mutations socioenvironnementales leur a permis de survivre et de continuer à s'épanouir après leur expulsion de ce parc dans les années 1970 - 1975. Des initiatives et autres facteurs endogènes et exogènes de renforcement l'expliquent. Cette résilience des communautés est d'une grande importance pour le développement (Matarrita-Cascante et al., 2017). Elle exprime une volonté des communautés de se prendre en charge et de contrôler leur développement par des stratégies réactives au changement (Wilson, 2012).

La meilleure compréhension de la résilience d'une communauté consiste à appliquer les principes écologiques à l'analyse des systèmes sociaux en fonction du relèvement et de la durabilité (Zautra et al., 2008). En intégrant ces deux propriétés, on a identifié trois éléments de résilience selon Folke et al. (2002) dont : (1) «l'ampleur du choc que le système peut absorber et rester dans un état donné;»(2) «le degré auquel le système est capable d'autoorganisation ; « et ( 3 ) » la mesure dans laquelle le système peut renforcer les capacités d'apprentissage et d'adaptation ». L'évaluation des progrès réalisés en matière de résilience requiert une attention particulière aux processus intermédiaires qui augmentent les chances de résilience (Zautra et al., 2008). Le test final des résultats de résilience survient lorsque la communauté est confrontée à un défi de taille.

\section{Regard sur les stress ou traumatismes historiques des peuples autochtones liés à leur expulsion des aires protégées}

Les expulsions des peuples autochtones des aires protégées qui leur a privé des ressources dont ils dépendaient dans leurs espaces naturels, ne datent pas d'aujourd'hui. Les événements qui ont entouré ces expulsions ont été traumatisants (Sandlos, 2008). Ils ont souffert notamment de la marginalisation (Colchester, 2003 ;Tauli-Corpuz, 2016), de la pauvreté, de la perte de leurs moyens de subsistance, d'insécurité alimentaire, d'exécutions extrajudiciaires, de la rupture de leurs liens avec leurs sites spirituels et d'une 
privation d'accès à la justice et aux voies de recours (Tauli-Corpuz, 2016). ERND ( 2017), ajoute l'errance dans le milieu d'accueil (le droit de jouissance durable des terres ne leur ayant pas été reconnu), la dispersion, les contraintes d'errer ici et là au gré des circonstances, un habitat précaire et incertain, une mauvaise santé, des activités économiques dérisoires incertaines ainsi qu'une instruction scolaire quasi absente.

En tant qu'indépendants sur leur propre territoire, de nombreux peuples indigènes ont été refoulés de leurs terres, expropriés par des organismes gouvernementaux au nom de la conservation (Colchester, 2004). Les cycles d'expulsion des peuples autochtones de leurs terres ancestrales datent de plus ou moins deux siècles (IUCN, 2005); mais les statistiques précises des personnes déplacées pour mettre en place les aires protégées dans le monde, font défaut. En Angleterre, au XIe siècle, la «nouvelle forêt » première chasse royale, a contraint le déménagement forcé de 2000 personnes pour le sport des rois. Les Shoshones du parc Yellowstone aux USA ont été expulsés et 300 personnes furent tuées au cours des affrontements en 1877. Près de 600000 peuples tribaux ont été déplacés par les gestionnaires des aires protégées en Inde (Edelblutte \& Gunnell, 2014) et 30000 autochtones expulsés de la réserve forestière de Kibale et de corridor de Gibier en Ouganda (Colchester, 2003). Plusieurs auteurs renseignent qu'en 1856 déjà, les Ogiek du Kenya avaient été expulsés de leurs terres ancestrales érigées en aire protégée, « la forêt de Mau ». En 1890, le Peuple Miwok de la Sierra Nevada en Californie (USA) connut le même sort dans l'actuelle Réserve Yosemite (Colchester, 2003). $86 \%$ des zones protégées en Amérique latine sont en fait habitées (Amend et Amend, 1992), cités par Colchester ( 2004). Cette vague se poursuivit jusqu'à notre époque. L'expulsion des Boschimans San et d'autres groupes d'habitants traditionnels du Botswana de l'actuelle « Réserve centrale de gibier » du Kalahari en 2002 figurent parmi les dernières têtes de proue de ces expulsions (Nelson \& Hossack, 2003).

En RDC, environ 6000 Twa ont été expulsés de force de leurs terres ancestrales au PNKB/RDC (Barume Kwokwo, 2003). Cette délocalisation afin de créer des parcs nationaux commence avec la période coloniale par le décret royal de 1925 créant le Parc National de Virunga et après l'indépendance du pays, avec la création du Parc National de Kahuzi - Biega (PNKB) vers les années 1970 - 1975 et celui de la Maiko en 1970.

\section{Vers un renforcement de résilience communautaire, la reconnaissance des droits des peuples autochtones par rapport à leur traumatisme}

Norris \& Stevens (2007) ont souligné que la résilience des communautés, et par conséquent le bien-être de celles-ci, reposait sur un réseau de capacité d'adaptation, en particulier le développement économique, le capital social, l'information et la communication, ainsi que la compétence 
communautaire. Au cours de la première décennie du XXIe siècle, une série d'événements a mené au renforcement et à la reconnaissance des droits des peuples autochtones et des communautés locales en tant qu'acteurs et dépositaires de droits et de savoirs relatifs aux aires protégées. Lors du IVe Congrès mondial sur les parcs nationaux et les aires protégées qui s'est tenu à Caracas, au Venezuela, en 1992, fut recommandée l'élaboration de politiques sur les aires protégées qui tiennent compte des intérêts des peuples autochtones, des pratiques coutumières liées aux ressources et les systèmes traditionnels de régime foncier. En octobre 1996, le Congrès mondial de la nature, réuni à Montréal, a adopté une résolution sur les « Principes et lignes directrices sur les peuples autochtones et traditionnels et les aires protégées ». Par ailleurs, l'Union mondiale pour la conservation de la nature (UICN) a adopté à Kinshasa, au cours de sa 12ème Assemblée générale, une résolution reconnaissant pour la première fois la nécessité de respecter les droits fonciers des populations autochtones lors de la création d'aires protégées (Lassana Koné \& Mukumba Pacifique, 2018).

L'évolution progressive de ce nouveau modèle de renforcement a continué avec le 5e congrès mondial sur le Parc (WPC5) en 2003 et les accords de Durban, et s'est poursuivi en 2004 à la Convention sur la Diversité Biologique avec la traduction des résultats du WPC5 en Programme de travail sur les aires protégées et en 2007 avec l'adoption de la Déclaration sur les droits des peuples autochtones. (Crawhall, 2018).

Ce renforcement a été constaté aussi par le Plan d'action pour la mise en œuvre de la Déclaration Universelle de l'UNESCO sur la Diversité culturelle à son point 14 , et adopté à sa $31^{\text {ème }}$ session tenue à Paris, pour engager les Etats à respecter et protéger les savoirs traditionnels, notamment ceux des peuples autochtones.

D'autres institutions internationales, entre autres la Banque Mondiale et l'Union Européenne, disposent de politiques de sauvegarde internes sur les peuples autochtones. C'est le cas de la résolution du Conseil de l'Union Européenne, sur les peuples autochtones de novembre 1998 ainsi que la Politique Opérationnelle 4.10 de la Banque Mondiale.

Le département de l'information de l'ONU en 2013 note que la reconnaissance des peuples autochtones dans les lois et les constitutions des pays reste une gageure. Mais certaines avancées notables ont été réalisées. En 2010, la République centrafricaine a été le premier pays africain à ratifier la Convention No. 169 de l'Organisation Internationale du Travail, qui concerne tout spécialement les droits des peuples autochtones et tribaux. En 2011, la République Démocratique du Congo est devenue le premier pays d'Afrique à adopter une loi spécifique portant promotion et protection des droits des populations autochtones. 
La constitution du Kenya reconnaît historiquement les groupes marginalisés, notamment les populations autochtones. La constitution du Cameroun fait également mention des peuples autochtones, et au Burundi, la constitution prévoit une représentation particulière des autochtones Batwa à l'Assemblée nationale et au Sénat.

À l'échelon régional, l'inscription des «droits des peuples » dans la Charte africaine des droits de l'homme et des peuples sert de point de départ à la prise en considération des populations autochtones en vertu de la Charte. Un rapport phare publié en 2003 par le Groupe de travail sur les populations/communautés autochtones de la Commission africaine des droits de l'homme et des peuples fournit une analyse de la situation des peuples autochtones en Afrique et constitue une pièce maîtresse pour l'étude théorique des peuples autochtones en Afrique.

Le cadre légal actuel de la République Démocratique du Congo ne fait pas mention explicite des peuples autochtones, mais certains textes réglementaires dans le secteur forestier les citent aux côtés des communautés locales.

Le Décret présidentiel 06/141 du 10 novembre 2006 portant nomination des membres de la Commission interministérielle de conversion des titres forestiers atteste à son article 2, point 11 que «Dans le cas de présence des Communautés autochtones parmi les communautés locales riveraines à proximité des titres visés, la Commission sera ouverte à un membre additionnel, représentant ces communautés autochtones. »

Le Décret $\mathrm{N}^{\circ} 09 / 40$ du 26/11/2009 du Premier Ministre congolais, portant création, composition et organisation de la structure de mise en œuvre du processus de Réduction des Emissions issues de la Déforestation et de la Dégradation des Forêts (REDD), en son article 5 sur la composition des membres du comité national REDD, a réservé un siège à un représentant des peuples autochtones.

Le projet de loi sur la protection et la promotion des droits des peuples autochtones en RDC soutenu par certains parlementaires regroupés au sein du Collectif Parlementaire pour la défense des Peuples Autochtones est en discussion à l'Assemblée Nationale.

Des rapports officiels des différents ministères qui, reçoivent des appuis financiers de la Banque Mondiale et de l'Union Européenne, parlent des peuples autochtones.

Les rapports intermédiaires sur la mise œuvre de la Convention relative à la Diversité Biologique en RDC du Ministère de l'Environnement, de la Conservation de la Nature et du Tourisme (MECNT, 1997, 2001,2005, 2009, 2014) présentent les avancées que fournit la République Démocratique du Congo quant à la mise en œuvre et le suivi de la stratégie et du plan d'action sur la biodiversité pour les collectivités autochtones. 
Pour décrypter la résilience, les autochtones Baka du Cameroun définissent la résilience par la rupture et le rebond, un ensemble de mots qui collent à leur quotidien. L'agriculture, la formation des jeunes, le promotions des activités génératrices de revenus, la forêt communautaire, bénéficient de l'appui des institutions de développement, du Plan de Développement des Peuples Pygmées (DPPP) lui-même soutenu par la Banque Mondiale et du collectif d'associations vouées à la cause des autochtones Pygmées sont autant de stratégies endogènes et exogènes de renforcement de la résilience chez les autochtones Baka (Ngono, 2019).

Pour les communautés autochtones de Babuluko, riverains du PNKB à l'Est de la République Démocratique du Congo dans sa partie de basse altitude, la coalition mondiale des forêts, IRCC ( 2018) identifie les droits d' accès et de la gestion durable des ressources naturelles en incluant l'initiative de la foresterie communautaire dans la zone autochtone Pygmée Babuluko comme initiative de résilience pour cette communauté.

Le but de cet article est d'identifier les initiatives des résiliences communautaires développées par les riverains autochtones Batwa dans le contexte de conflit, dans la partie haute altitude du Parc National de Kahuzi Biega.

\section{Méthodes et matériels \\ Description de la zone d'étude}

Le PNKB a été créé pendant la période coloniale. Il s'étend du bassin du fleuve Congo près d'Itebero-Utu jusqu'à sa frontière occidentale au nordest de la ville de Bukavu. Il possède une faune et une flore riches, dont les représentants les plus spectaculaires sont les gorilles de plaine de l'Est. 524 espèces de gorilles sont endémiques à la RD. Congo et le PNKB est son dernier sanctuaire (Freddy et al., 2018).

Vaste de $6000 \mathrm{~km}^{2}$, le PNKB est situé à l'Est du Congo. Ses coordonnées géographiques sont les suivantes : entre $1^{\circ} 36^{\prime}-2^{\circ} 37^{\prime}$ de latitude Sud et $27^{\circ} 33^{\prime}-28^{\circ} 46^{\prime}$ de longitude Est. Le PNKB tire son nom des deux montagnes qui dominent sa partie haute. Ce sont les montagnes Kahuzi avec une hauteur de $3308 \mathrm{~m}$ et les montagnes Biega avec une altitude de $2790 \mathrm{~m}$. C'est cet ensemble de beautés naturelles extraordinaires, riches et diversifiées en termes de biodiversité et de culture qui a fait de ce merveilleux parc national, un site du patrimoine mondial de l'UNESCO depuis 1980. 


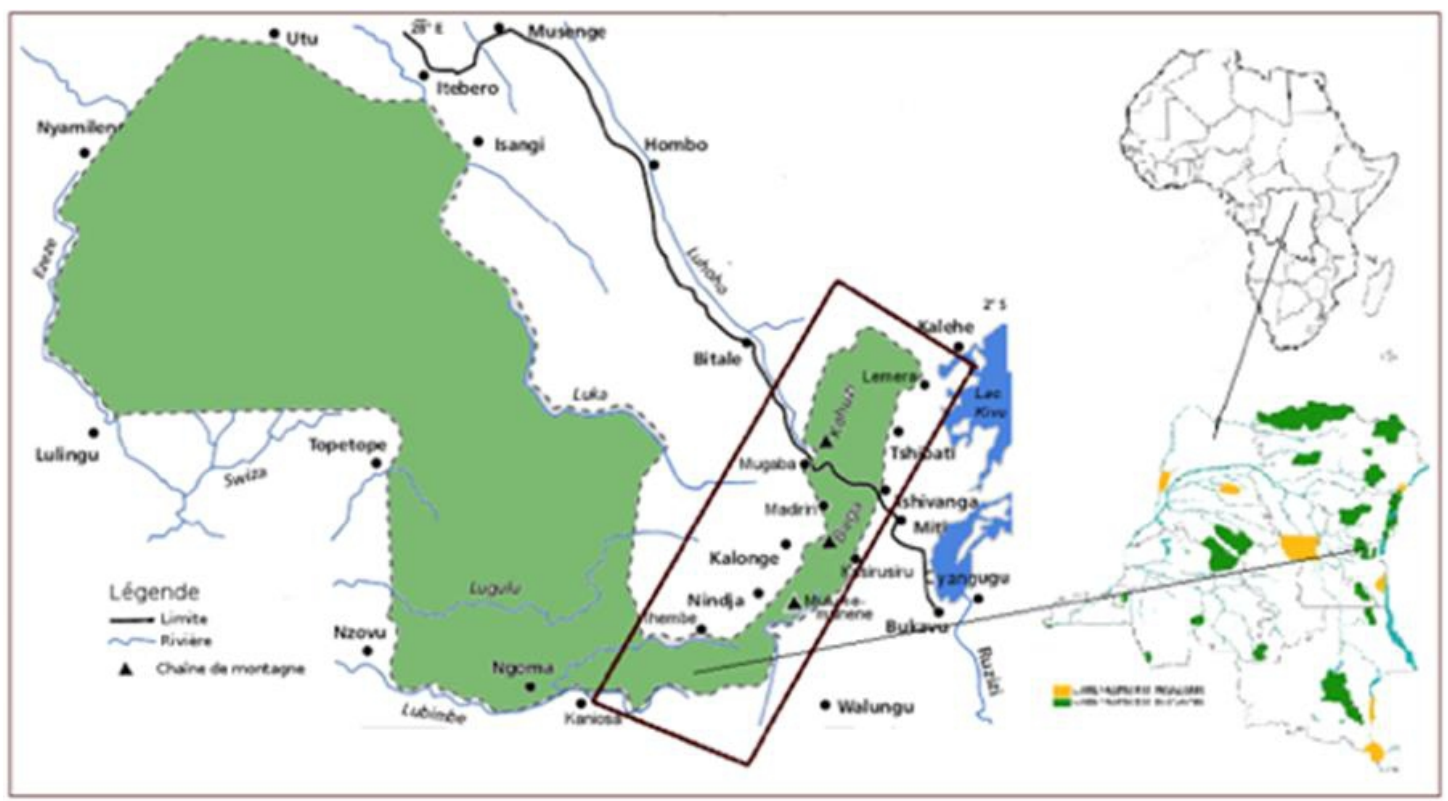

Figure. 1 : Carte du PNKB et sa localisation en RD. Congo

\section{Collecte des données}

L'étude étant qualitative, sur le terrain, la récolte des données a été effectuée grâce à un guide d'entretien pour favoriser le caractère évolutif des données et le contact direct avec les participants (Sylvain, 2002). Des interviews approfondies ont été réalisées avec des personnes ressources censées détenir des informations nécessaires pour cette étude. L'entretien a été fait séparément d'abord avec les leaders autochtones Batwa riverains, mais aussi avec les représentants autochtones Batwa riverains dans les trois sites de l'étude à savoir l'axe Kabare, l'axe Kalehe-Ihusi, l'axe Bunyakiri-Bitale.

Des groupes focalisés, des interviews personnalisées, des ateliers brainstorming, ont été réalisés. Au total, six discussions de groupe ont été organisées à raison de trois discussions par axe de recherche. Les focus groupes étaient constitués chaque fois de dix hommes, dix femmes et un groupe de 10 jeunes à raison de 5 filles et 5 garçons. Ces groupes de discussions nous ont ainsi permis de confronter les propos recueillis auprès des personnes ressources afin de déceler les divergences et les convergences intergroupes. Dans leur quasi-totalité, nos enquêtés sont constitués d'analphabètes âgés de 15 à plus de 65 ans et ont comme principales activités l'agriculture et le petit commerce ainsi que des travaux journaliers.

Les données ont été complétées par la documentation des résultats de recensement réalisé par le Centre d'accompagnement des Autochtones Pygmées et Minoritaires Vulnérables «CAMV» avec l'accompagnement méthodologique de l'Institut national de statistique de la République Démocratique du Congo, division provinciale du Sud Kivu à Bukavu en 2016. 
Dans les groupes focalisés, nous avons invité d'abord les femmes, puis les hommes, à classer l'impact de chaque perturbation sur leur moyen de subsistance, de survie... C'est à dire à découper les données qu'ils disposent en unités de sens puis procéder à leur regroupement pour former des catégories (Allard-Poesi, 2011), en utilisant des signes ( +++ ou à ++++ ) pour représenter les pratiques moyennes, élevées et des signes $(+\mathrm{ou}++)$ pour représenter les pratiques résilientes très faibles ou faibles. Plus une action est pratiquée, il lui est attribué de signes plus, jusqu'à quatre maximum.

\section{Résultats}

A la suite de leur expulsion, les Batwa riverains se sont installés sur des terres des populations bantoues sans terre car aucune mesure d'accompagnement n'a été mise en place. Ils ont toujours occupé les terres à titre précaire octroyées par les chefs coutumiers sur des sites d'accueil et ils sont obligés parfois de se déplacer constamment, ce qui constitue une véritable insécurité et une situation de stress permanent. Cette situation d'errance était accentuée par les guerres en répétition que l'Est de la République Démocratique du Congo a connues. Ce tableau 1 présente la répartition des riverains Batwa recensés selon qu'ils ont été expulsés ou pas dans le Parc National de Kahuzi - Biega

Tableau 1. Répartition des riverains Batwa recensés expulsés et non expulsés dans la partie haute altitude de l'hinterland du Parc National de Kahuzi - Biega

\begin{tabular}{|c|c|c|c|c|}
\hline $\begin{array}{l}\text { EXPULSION } \\
\text { PNKB }\end{array}$ & $\begin{array}{c}\text { BUHAVU } \\
\text { (Kalehe) } \\
\mathbf{n}=7990\end{array}$ & $\begin{array}{c}\text { BULOHO } \\
\text { (Kalehe) } \\
\text { n=683 }\end{array}$ & $\begin{array}{c}\text { KABARE } \\
\mathbf{n}=935\end{array}$ & $\begin{array}{c}\text { Total des riverains } \\
\text { Batwa } \\
\text { n=9608 }\end{array}$ \\
\hline EXPULSES & $8 \%$ & $5 \%$ & $8 \%$ & $7 \%$ \\
\hline NON EXPULSES & $92 \%$ & $95 \%$ & $92 \%$ & $93 \%$ \\
\hline
\end{tabular}

Source : Recensement effectué par l'organisation CAMV en 2016 avec l'accompagnement méthodologique de l'Institut National de la Statistique.

Ce tableau 1 renseigne que dans l'ensemble, seulement $7 \%$ de riverains autochtone Batwa recensés sont des survivants des expulsions de leurs terres ancestrales devenues aire protégée.

\section{Eléments actuels qui conduisent les Batwa riverains au traumatisme dans la partie haute altitude du Parc National de Kahuzi Biega (PNKB)}

Avant leur expulsion du PNKB, les riverains autochtones Batwa étaient des nomades, cueilleurs, chasseurs... L'expulsion les a obligés de changer de mode de vie et de perdre les moyens de leur subsistance pour s'adapter à une nouvelle vie dans l'hinterland du parc. Ils sont obligés de devenir sédentaires et de se convertir en agriculteurs, éleveurs... Les éléments de traumatisme et chocs subis par ces riverains autochtones Batwa sont entre 
autres, l'absence de terres communautaires, la perte des forêts où ils peuvent exercer les rites traditionnels, l'absence des forêts où ils peuvent pratiquer la chasse, la cueillette, le ramassage et la récolte des produits forestiers non ligneux, la mortalité et la morbidité élevées faute d'argent, le non-accès aux ressources du parc entre autres plantes médicinales, les décès et arrestations par les éco gardes de l'ICCN.

\section{Les initiatives de résiliences des riverains Batwa dans le contexte de conflit au PNKB}

Suite au stress consécutif à leur expulsion brutale vers les années 19701975 des forêts de Kahuzi - Biega de haute altitude qu'ils occupaient, ils ont pu développer des pratiques résilientes pour s'adapter à la nouvelle vie imposée. Certaines pratiques ou initiatives sont anthropologiques.

Les riverains autochtones Batwa de la partie haute altitude ont des vocabulaires particuliers. Certains mots ou groupes de mots sont utilisés pour l'adaptabilité et l'encouragement dans leur milieu. Il s'agit entre autres :

$\Rightarrow$ Omuchwa arharhama = l'autochtone Pygmée ne se fatigue pas.

$\Rightarrow$ Omurhwa akakusima wana cirha = celui qui est aimé par l'autochtone Pygmée est heureux.

$\Rightarrow$ Omurhwa akakugaya orhakacigera ahali = Lors que tu es haï par l'autochtone Pygmée, tu ne peux plus franchir là où il est.

$\Rightarrow$ Omurhwa ye mwami $=\mathrm{L}$ ' autochtone Pygmée est le roi.

$\Rightarrow$ Omurhwa arhabarhula $=$ L'autochtone Pygmée ne transporte pas des fagots.

$\Rightarrow$ Omurhwa orhashigira arhafuma i'shali = un autochtone Pygmée qui ne grimpe pas n'échappe pas à la faim!

Par contre, d'autres initiatives de résiliences communautaires sont d'ordre interne ou culturellement reconnues ou développées d'une manière propre aux riverains autochtones Batwa. D'autres sont externes ou renforcées de l'extérieur.

Le tableau. 2 donne selon l'importance, les principales initiatives de résilience des riverains Batwa répertoriées. 
Tableau 2. Les principales initiatives de résilience des riverains Batwa

Initiatives résilientes

$\Rightarrow$ Dialogue et concertation avec les gestionnaires du parc avec la participation d'autres parties prenantes

$\Rightarrow$ Connaissance de leurs droits et leurs revendications.

$\Rightarrow$ Education formelle et alphabétisation

$\Rightarrow$ Cohésion et cohabitation avec les Bantous et autres communautés non Pygmées.

$\Rightarrow$ Accès à l'eau potable

$\Rightarrow$ Amélioration de l'habitat

$\Rightarrow$ Disposition /possession de lopins de terre par certains ménages

$\Rightarrow$ Amélioration du mode d'habillement.

$\Rightarrow$ Amélioration de la quantité de soins offerts aux Batwa.

$\Rightarrow$ Exercices des activités agricoles

$\Rightarrow$ Activité culturale et vente de chanvre "Cannabis sativa"

$\Rightarrow$ Recours au petit élevage (cobayes et chèvres)

$\Rightarrow$ Entrée clandestine dans le PNKB (Recours au Parc pour récolter les PFNL)

$\Rightarrow$ Espoir aux promesses faites par le Parc

$\Rightarrow$ Mendicité, et vols dans les champs de leurs voisins Bantous

$\Rightarrow$ Exercice des travaux de cash for work (Rukotwa/ Homme/Jour)

$\Rightarrow$ Engagement des Batwa au PNKB

$\Rightarrow$ Ramassage (après récolte chez les voisins)

$\Rightarrow$ Recours/domestication d'espèces sauvages

$\Rightarrow$ Recours à la pharmacopée /collectes de plantes sauvages

$\Rightarrow$ Appui des autorités locales, provinciales, nationales et coutumières.

Légende : + pratiques très faible $;++$ pratiques faibles, ++++ Pratiques élevées.
Très faible Faible Moyenne

Elevée

$+++$

$+++$
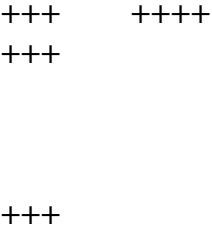

$++$

$++$

$+$

$+$

$+$

$+$

$+$

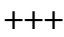

$++++$

$+++$
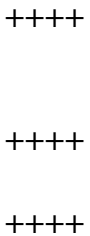

$++++$

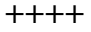

$++$

$+++$

+++ pratiques moyennes, 
Au regard du tableau 2, vingt-trois (23) pratiques résilientes ont été identifiées dont les pratiques résilientes endogènes (1) possédant «++++» viennent à la première position. Ces actes sont ceux pratiqués et provenant des communautés riveraines autochtones Batwa. Elles ont eu un impact significatif dans leur adaptabilité et leur durabilité depuis leur expulsion de la partie haute altitude du Parc National de Kahuzi Biega. Les pratiques résilientes exogènes (2) possédant « +++ », sont celles provenant de l'extérieur et viennent en seconde position.

Parmi ces initiatives on distingue :

Les initiatives endogènes de résilience des riverains autochtones Batwa dans le contexte de conflit au PNKB

Entrée clandestine dans le PNKB et recours au PNKB pour collecter les produits forestiers non ligneux et autres concessions forestières dans l'hinterland du PNKB.

La chasse et la récolte des produits forestiers non ligneux «PFNL » contribuent à la survie des ménages riverains dans les trois sites. En effet, cette pratique est prohibée et dangereuse car les zones forestières qui entourent les villages autochtones enquêtés constituent une partie entière du Parc National de Kahuzi - Biega à savoir la concession forestière de l'Institut National pour 1' Etudes et la Recherche Agronomique (INERA) à Bitale et quelques réserves forestières des fermiers à Bunyakiri. Les riverains autochtones Batwa pénètrent dans ces concessions pour pratiquer des activités de survie comme la chasse, la cueillette des champignons, la recherche du miel, la collecte des rotins et les fils de l'igname sauvage " Dioscorea prehensilis" qui leur servent dans la fabrication des paniers.

L'axe de Buziralo excelle par contre dans la fabrication des paniers, des vannes, des pots en terre cuite, des ruches traditionnelles, des malaxeurs, des mortiers, des pilons, etc. Il se pose malheureusement encore une fois de plus le problème d'accès aux matières premières pour la fabrication de ces œuvres d'art qui sont devenus difficilement trouvables. Certains autochtones Batwa riverains courageux font deux jours de voyage pour s'en procurer vers Bunyakiri à plus ou moins dix ou quinze kilomètres.

Un constat général s'est dégagé des discussions avec les Pygmées : la fabrication des œuvres d'art requiert plusieurs heures de travail et rapporte moins d'argent. Les Pygmées préfèrent ainsi s'adonner au petit commerce et à des travaux journaliers dans les champs des Bantous afin d'assurer leur survie. Il faut souligner aussi que c'est une petite poignée d'entre eux qui fabrique ces œuvres d'art en l'occurrence les paniers qu'ils revendent à 2,5\$ la pièce après trois à quatre jours de travail, sans compter les jours consacrés à la recherche des matières premières. 


\section{Collecte et domestication des plantes sauvages par les Pygmées}

Les riverains Batwa autochtones exploitent certaines espèces sauvages ex-situ. Les principales cultures qu'ils domestiquent sont notamment des ignames sauvages "Birongo », des ignames « chuhuko », des ananas sauvages byahambahamba, mahaliya, mawu,ngobia, des légumes « huha », des carottes sauvages mitero, des champignons mbumba, buhwa, kashalulabahinvi, burobi, ndelama, bwandwa, birerabana, mangaka, bunombe, lulako, birembo, etc.

Pour éviter l'attrait des riverains Batwa vers le Parc National de Kahuzi - Biega, certaines solutions ont été envisagées par eux-mêmes et parfois appuyées par certaines organisations locales qui les accompagnent pour leur autopromotion. C'est notamment la domestication de certaines espèces sauvages priorisées dans le tableau 3 ci-après :

Tableau 3. Dix espèces sauvages priorisées pour la domestication par les Pygmées

$\begin{array}{lll}N^{\circ} & \text { Nom local de la plante } & \text { Nom scientifique de la plante } \\ \text { 1. } & \text { Bihumyo } & \text { Termitomyces } s p . \\ \text { 2. } & \text { Birongo } & \text { Dioscorea minutiflora } \\ \text { 3. } & \text { Byahambahamba } & \text { Myrianthus arboreus } \\ \text { 4. } & \text { Chibirabira } & \text { Neoboutonia macrocalyx } \\ \text { 5. Huha } & \text { Solanum indicum } \\ \text { 6. } & \text { iyange } & \text { Dioscorea bulbifera } \\ \text { 7. } & \text { Mawu } & \text { Strycnos innocua } \\ \text { 8. } & \text { Masinza ou chihuku } & \text { Dioscorea alata } \\ \text { 9. } & \text { Mitunguru } & \text { Aframomum gilleti } \\ \text { 10. Ngobia } & \text { Dacryoides edulis }\end{array}$

Il s'est dégagé que les Batwa riverains souhaitent domestiquer certaines plantes sauvages qu'ils trouvaient jadis dans la forêt. Il se trouve malheureusement qu'il se pose un problème de semences/boutures car ils n'ont plus accès à la forêt. Seul le Birongo (Dioscorea) est accessible même en dehors du Parc. Ils font recours pour le reste à l'entrée clandestine dans le parc ou dans d'autres concessions forestières privées dans l'hinterland du parc. Le manque de terres à cultiver et l'accès difficile aux tuteurs de la culture de Birongo constituent l'obstacle principal à cette activité.

\section{Mendicité et vols dans les champs des voisins non autochtones Batwa}

Les riverains autochtones Batwa mènent une vie d'errance, de mendicité, dépourvue de toute dignité humaine. Réduits à la situation des « paysans sans terres », ils vivent à la merci des populations hôtes qui les emploient dans une forme de servitude moyenâgeuse et, clochardisés, ils vivent dans une spirale de « servitude pour dettes » pourvu qu'ils survivent. 
Ce manque d'habitude fait que certains riverains Batwa pour survivre et suppléer aux besoins des ménages, s’adonnent aux vols des récoltes de leurs voisins directs “Bashi, Havu et Tembo " d'où les conflits fréquents dus aux vols des récoltes dans les champs.

\section{Ramassage (après récolte chez les voisins) et exercice des travaux de cash for work (Rukotwa/ Homme/Jour)}

Les riverains autochtones Batwa sont contraints à fournir leur force physique dans les travaux de champs, à vendre les produits du ramassage tels que les produits oubliés ou laissés dans les champs d'autrui après les récoltes, les chenilles et les champignons récoltés dans des plantations, fermes ou concessions forestières des privés dans l'hinterland du parc, ou vendre de menus instruments issus de leur travail d'artisanat mais tout cela à vil prix. Ils servent aussi comme gardes, pisteurs et guides dans le parc.

\section{Espoir aux promesses données par le parc}

Les riverains autochtones Batwa affirment que le parc leur donne toujours des promesses qui auraient dû être honorées par le gouvernement congolais depuis leur expulsion. Cependant, ils gardent toujours espoir suite au dialogue qu'ils engagent avec les gestionnaires du parc mais également, ils espèrent que le procès qu'ils ont intenté contre le parc pourra aboutir et ils espèrent obtenir gain de cause. Les promesses données vont souvent dans le sens de trouver du travail et être engagé d'une manière permanente au parc, de partager des ressources issues de la gestion du parc, d'accéder aux structures de base, d'accéder également aux ressources du par cet à la terre.

\section{Activité culturale et vente de Cannabis sativa.}

Le chanvre «Irumba » «Cannabis sativa » est toujours présent dans les villages des riverains autochtones Batwa et partout où ils se retrouvent dans l'hinterland du parc. Selon eux, c'est un stimulant à l'instar du café. Les riverains autochtones Batwa estiment que le chanvre soigne beaucoup de maladies entre autres, le diabète, les maux d'estomac, la rougeole etc. Et tout toxicomane qui consomme régulièrement le Cannabis sativa ne pourrait contracter le «VIH/Sida».

Quand ils n'ont rien d'autres comme activités à réaliser, ces Batwa, aussi bien les hommes que les femmes en prennent à volonté pour leur plaisir. "Quand nous en prenons, nous vivons l'extase, nous oublions les traumatismes et nous nous construisons un avenir meilleur dans l'esprit», estiment-ils. Ils le vendent également ou le troquent contre d'autres produits chez les voisins, y compris même les policiers et les militaires. 


\section{Les initiatives exogènes de résilience des riverains autochtones Batwa dans le contexte de conflit au PNKB}

\section{Les pratiques agricoles}

L'agriculture et l'élevage ressortent comme des activités très importantes de survie pratiquées avec l'appui externe des institutions d'appui aux riverains autochtones Batwa dans l'hinterland du PNKB. Cela s'expliquerait par le fait que les ménages de ces Batwa ont bénéficié des appuis en élevage de la part des associations accompagnatrices et autres institutions étatiques. Le Centre d'accompagnement des autochtones Pygmées et Minoritaires vulnérables «CAMV » et le Parc National de Kahuzi - Biega leur ont distribué des géniteurs de chèvres. Le CAMV continue à distribuer les semences de haricot et arachide aux ménages des quelques villages pilotes de Bitale à Bunyakiri, Chombo et ses alentours dans Kabare et Buziralo et leurs alentours dans le Kalehe.

Les riverains Batwa ont appris l'agriculture auprès d'autres peuples riverains du Parc de Kahuzi-Biega après leur expulsion de la forêt érigée en parc actuellement. Cette pratique agricole pour les riverains autochtones est limitée du fait qu'ils ne possèdent pas des terres sur lesquelles ils peuvent exercer leur droit. Pour les voisins des riverains Batwa (les Tembo, les Shi et les Havu), le droit d'usage de la terre est héréditaire et se transmet de père en fils. Ce qui n'est pas le cas pour les Batwa qui ont été expulsé du parc. Les riverains Batwa qui désirent acquérir un lopin de terre de la part du chef de village en fermage pour y exercer l'agriculture ou pour y construire une maison, doivent payer des redevances (Kalinzi). Malheureusement faute des moyens, ils prennent en location des lopins de terre très réduits juste pour la petite agriculture qui n'arrive pas à subvenir à leurs besoins en nourriture. Pour remédier tant soit peu à ce problème de manque de terre, certaines organisations locales qui les accompagnent, prennent en location ou achètent des terres qu'ils leur donnent pour la construction des petites maisons et pour l'exploitation des champs communautaires aux fins de la domestication de certaines plantes sauvages.

\section{Les pratiques des petits élevages}

La pratique du petit élevage ne rentre pas également dans les habitudes traditionnelles des riverains autochtones Batwa. Néanmoins, ne pouvant plus pratiquer la chasse dans la forêt devenue aire protégée, ils pratiquent l'élevage à faible échelle. Ils s'adonnent ainsi à l'élevage du petit bétail et de la bassecour pour se procurer tant soit peu des protéines d'origine animale qu'ils trouvaient jadis dans la chasse des animaux sauvages. L'élevage est pratiqué dans tous les sites des riverains autochtones Batwa. La figure 2 montre les types d'élevage pratiqués par les riverains Batwa dans la partie haute altitude du Parc National de Kahuzi Biega. 


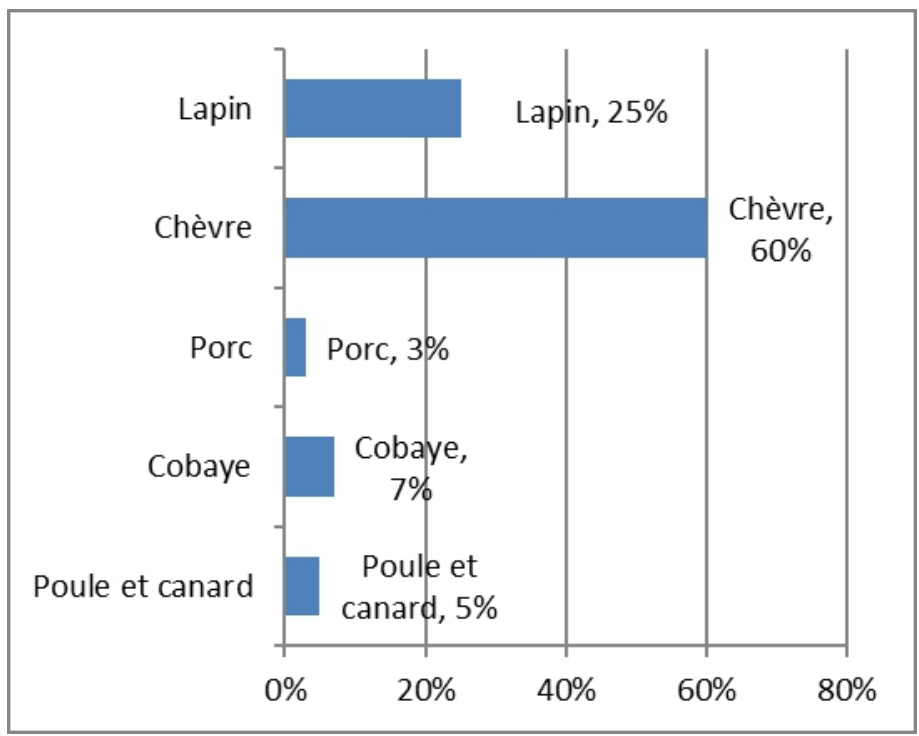

Figure 2. Quelques types de petit élevage pratiqués par les autochtones Batwa dans l'hinterland du PNKB

Il ressort de la figure ci-dessus, que les préférences des Pygmées visà-vis de l'espèce du type d'animal à élever sont de l'ordre de $60 \%$ pour les chèvres, suivis par les lapins à hauteur de $25 \%$, les cobayes $7 \%$, les poules et les canards 5\%, et les porcs 3\%. Nous n'avons cependant pas trouvé un seul Pygmée qui pratique l'élevage du gros bétail. Les raisons avancées sont le manque de ressources financières suffisantes pour payer les géniteurs en plus des contraintes d'accès à la terre. Certains d'entre eux voudraient s'investir dans l'élevage de bovins mais trouvent que cette activité exige trop d'efforts en termes de temps pour paitre les vaches et la reproduction des vaches prend beaucoup de temps avec neuf de gestation. L'élevage du gros bétail reste ainsi, l'apanage de leurs voisins directs Shi, Tembo et Havu et leur confère une supériorité en termes des ressources financières et de prestige ou de considération sociale vis-à-vis des Pygmées.

Les Pygmées interrogés affirment qu'ils préfèrent la chèvre car elle représente une valeur économique considérable (plus ou moins 50 dollars US) et est facile à élever. Le porc (plus ou moins 100 dollars US), est très prolifique mais est plus exigeant en termes d'alimentation, ce qui explique son faible score en dépit de sa valeur économique élevée. Le cobaye est prisé pour son caractère aussi très prolifique, la facilité de son élevage constitue une réponse au problème d'alimentation des Pygmées et d'accès aux petites ressources.

Il sied tout de même de relever que des contradictions s'observent par rapport aux raisons évoquées par les Pygmées quant au choix à opérer entre le cobaye et la chèvre. Ces derniers reconnaissent le caractère très prolifique du cobaye et la facilité de l'abattre pour subvenir au besoin d'alimentation et 
d'autres besoins primaires. Ceci n'est pas le cas par contre pour la chèvre car on ne l'abat pas facilement pour des besoins d'alimentation ou d'autres besoins primaires. Seuls des cas de force majeure ou d'extrême urgence, amènent les Pygmées à abattre ou à vendre la chèvre.

L'élevage est une activité qui peut être envisagée comme une alternative pour sauvegarder la faune sauvage pour une exploitation plus rationnelle par les générations aussi bien présentes que futures.

\section{Pêche}

Quelques rares personnes enquêtées ont reconnu avoir passé quelques moments dans les zones côtières du lac Kivu où elles ont pratiqué la pêche artisanale dénommée dans la langue locale «Kurura », et la capture par cette forme de pêche est très moindre.

\section{Renforcement des capacités en dialogue et droits des peuples autochtones.}

Un processus de dialogue constitué d'une série d'évaluations dit «Whakatane » est en cours au Parc National de Kahuzi - Biega (PNKB) depuis 2014. Le processus est axé sur le règlement pacifique des différends entre les riverains autochtones Batwa et le parc. En 2014, les communautés autochtones Batwa, le PNKB, l'organisation nationale Centre d'Accompagnent des autochtones Pygmée et Minoritaires Vulnérables «CAMV», le gouvernement et parlement provinciaux et d'autres parties prenantes ont élaboré une feuille de route dite de Whakatane pour parvenir à garantir l'accès des riverains Batwa aux ressources naturelles du parc entre autre la terre mais aussi la santé, l'éducation et l'emploi. Il s'est agi aussi d'assurer un partage des bénéfices découlant de la gestion du parc. Le processus est appuyé par des expériences des Ogiek du Mont Elgon du Kenya qui sont aussi dans le même processus.

Mises à part les sessions de formation pour la promotion des droits des riverains autochtones Batwa appuyées par les ONG, un groupe d'autochtones Batwa a jugé aussi bon d'intenter un processus contre le parc depuis 2008 et l'affaire est toujours à la cour de cassation.

\section{Discussion}

Les riverains autochtones Batwa ayant été expulsés du Parc National de Kahuzi - Biega (PNKB), se sont retrouvés dispersés dans divers villages d'accueil dans l'hinterland du parc. Ils étaient obligés d'adopter un mode de vie issu de certaines initiatives endogènes et exogènes de résilience réalisées pour la durabilité du développement du milieu dans lesquels ils évoluent. La durabilité du développement dépend des pratiques endogènes et des intrants exogènes de soutien, constante Badal (2015, 2018). 
Cependant, pour le cas des riverains autochtones Batwa dans la partie haute altitude du PNKB, les initiatives endogènes ont eu un impact significatif dans leur mode de vie que les initiatives exogènes de résilience. Toutefois, certaines initiatives endogènes de résilience pour les riverains Batwa sont dangereuses, contraires aux lois du pays. Elles ne se réalisent pas sans casse et mort d'homme. Le Plan Général de Gestion 2009-2019 (PGG/PNKB 20092019) montre que les riverains autochtones Batwa recourent à la vente et au troc du Cannabis sativa, du bois et de la viande de brousse. Le PGG/PNKB 2009-2019 estime que ce mode actuel d'exploitation illégale des ressources du PNKB par les Batwa représente un grand risque pour l'avenir de celui-ci, car ils entrent clandestinement dans le parc à la recherche de moyens de subsistance. A ce sujet Matabaro ( 2009), écrit que les Pygmées, peuples non sédentarisés et vivant parfois à l'intérieur des forêts classées, sont quotidiennement en contradiction avec la loi sur la conservation de la nature car celle-ci interdit sans réserve toute activité humaine à l'intérieur des aires protégées. Ils sont conscients que la réglementation en vigueur le leur interdit (ERND, 2017) et sont souvent en altercation avec les agents de l'Institut Congolais pour la Conservation de la Nature commis à la garde du Parc National de Kahuzi - Biega (PNKB). Pour eux, aucune vie n'est possible sans la forêt. Ils prennent toutes les matinées de sérieux risques en entrant clandestinement dans le PNKB (Lyamahesana, 2013).

L'étude socio-économique et culturelle, attitudes et perceptions des communautés Twa pygmées du secteur Mikeno/ Secteur Sud du Parc National des Virunga réalisée par la Banque mondiale aux alentours de cette aire protégée renseigne que pour leur survie, les Batwa riverains du Parc National de Virunga pratiquent des activités agricoles et économiques pour leur survie mais se limite juste à la résolution du problème de leur alimentation. Ils vivent donc au jour le jour, sans penser au lendemain. Ainsi comme le montre Ngono ( 2019), les stratégies de résilience des autochtones sont élaborées entre autres à partir de l'intégration à la vie moderne et à l'assimilation des mécanismes d'adaptation au mode de vie des Bantous, à leur habitat, à leur scolarisation, etc. aux pratiques d'agriculture (Colchester, 2003 ; Folke et al., 2016 ; Ngono, 2019), aux pratiques du petite élevage et autres appuis en micro-crédit rotatifs avec le soutien des organisations nationales et internationales (Folke et al., 2016). La mobilisation de l'État et de la société civile à travers des projets de développement des villages pygmées, les accompagne vers une résilience positive.

Pour renforcer la résilience des mutations socio- environnementales au sein d'une communauté de chasseurs/cueilleurs, les Etats et les organismes nationaux et internationaux doivent réaliser des vastes programmes de développement durable, estime Ngono (2019). 
Le renforcement de la résilience nécessite une approche intégrée et un engagement à long terme pour améliorer trois capacités critiques: la capacité d'absorption, la capacité d'adaptation et la capacité de transformation (Béné et al., 2012). Les initiatives de survie auxquelles s'adonnent les riverains autochtones Batwa dans le milieu d'accueil, sont justes à classer dans leur capacité d'adaptation. Par contre, leur capacité de transformation est loin d'être aboutie car cela concerne les mécanismes de gouvernance, les politiques/réglementations, l'infrastructure, les réseaux communautaires et les mécanismes formels de protection sociale qui font partie du système plus vaste dans lequel les communautés sont intégrées (Béné et al., 2012).

Ainsi l'approche de résilience socio-écologique sera réalisée en mettant davantage l'accent sur le développement pour le bien-être humain tout en prenant en compte le contexte de la biosphère et en relation avec la durabilité. Ici la capacité d'adaptation fait référence aux actions humaines qui soutiennent, innovent et améliorent le développement sur les voies actuelles (Folke et al., 2016).

\section{Conclusion}

Cette étude visait à identifier les initiatives de résiliences communautaires développées par les riverains autochtones Batwa dans le contexte de conflit, dans la partie haute altitude du Parc National de Kahuzi Biega.

Vingt-trois (23) initiatives résilientes communautaires ont été identifiées dont cinq (5) sont endogènes et dix-huit (18) exogènes. Les initiatives endogènes de résilience ont eu plus d'impact significatif dans le mode de vie des riverains autochtones Batwa comparées aux initiatives exogènes de résilience.

Les riverains Batwa sont devenus des paysans sans terre qui cultivent pour les autres à vil prix. Ils vont chercher des plantes médicinales et du miel dans le parc ou concessions forestières privées périphériques et prennent parfois les récoltes des voisins sans leur autorisation. Le PNKB leur a promis un accompagnement adéquat qui tarde toujours à venir. Dans leur milieu d'accueil, les initiatives de résiliences communautaires qu'ils développent sont juste à classer dans leur capacité d'adaptation et non de transformation. La transformation exige des mécanismes de gouvernance, des politiques / réglementations, de l'infrastructure, des réseaux communautaires et des mécanismes formels de protection sociale qui font partie du système plus vaste dans lequel les communautés sont intégrées. Des lois existent mais méritent d'avoir des textes d'application spécifiquement pour le volet encadrement des riverains autochtones Batwa expulsés des parcs en République Démocratique du Congo. La promotion et le renforcement des initiatives de résiliences communautaires endogènes compatibles avec la législation nationale ainsi que 
celles exogènes avec un focus sur les moyens de subsistance et l'accès aux structures sociales de base pour ces Batwa s'inscrivent dans les actions durables pour la sortie de la vulnérabilité de ce groupe.

\section{References :}

1. Allard-Poesi, F. (2011). Le codage n'est pas un «truc» méthodologique ou du codage comme «problématisation». Le libellio d'Aegis, 7(3), 38.

2. Badal, B P. (2015). Debate on Exogenous and Endogenous Rural Development. Nepalese Journal of Development and Rural Studies, 15-22.

3. Badal, Bharat Prasad. (2018). Alternative rural development: Social Justice, Equity, Self-Reliance, and Third Worldism. Research Nepal Journal of Development Studies, 1(1), 1-17.

4. Barume Kwokwo, A. (2003). En voie de disparution? Les droits des autochtones en Afrique: Le cas des Twa du parc national de Kahuzi Biega, en République du Congo.

5. Béné, C., Wood, R. G., Newsham, A., \& Davies, M. (2012). Resilience: New utopia or new tyranny? Reflection about the potentials and limits of the concept of resilience in relation to vulnerability reduction programmes. IDS Working Papers, 2012(405), 1-61.

6. Borrini-Feyerabend, G., Dudley, N., Jaeger, T., Lassen, B., Broome, N. P., \& Phillips, A. (2014). Gouvernance des aires protégées : De la compréhension à l'action. Collection des lignes directrices sur les meilleures pratiques pour les aires protégées No. 20. Gland: UICN.

7. Colchester, M. (2003). Nature sauvage, nature sauvée? Peuples autochtones, aires protégées et conservation de la biodiversité. World Rainforest Movement.

8. Colchester, M. (2004). Conservation policy and indigenous peoples. Environmental Science \& Policy, 7(3), 145-153.

9. Crawhall, N. (2018). Les influences sociales et économiques $s$ ' exerçant sur les aires protégées. Worboys, GL, Lockwood, M., Kothari, A., Feary S. et Pulsford I.(éd ....

10. Edelblutte, É., \& Gunnell, Y. (2014). Le territoire impossible des populations tribales du parc national Sanjay Gandhi (Mumbai, Inde). LEspace geographique, 43(1), 1-17.

11. ERND. (2017). L'expulsion des populations Pygmees du Parc National de Kahuzi-Biega: Faits, conséquences et perspectives. Rapport d'étude. ERND Institute, ASBL. R.D.CONGO. 
12. Folke, C., Biggs, R., Norstrom, A. V., Reyers, B., \& Rockstrom, J. (2016). Social-ecological resilience and biosphere-based sustainability science.

13. Folke, C., Carpenter, S., Elmqvist, T., Gunderson, L., Holling, C. S., \& Walker, B. (2002). Resilience and sustainable development: Building adaptive capacity in a world of transformations. AMBIO: A journal of the human environment, 31(5), 437-441.

14. Freddy, M. B., Jacques, F. Z., \& Balola, K. (2018). Aménagement d'une zone tampon autour du Parc National de Kahuzi-Biega (PNKB) en haute altitude (Nord-Est) à Bugorhe et Irambi-Katana, dans le Territoire de Kabare, Sud-Kivu, RD Congo. International Journal of Innovation and Applied Studies, 23(4), 523-540.

15. IRCC, C. mondiale des forêts. (2018). Initiative de conservation et resilience communautaire. Ronnie Hall.

16. IUCN. (2005). Plan d'action de Durban, version révisée, mars 2004.

17. Lassana Koné, \& Mukumba Pacifique. (2018). La Conservation et les droits des communautés locales et des peuples autochtones en République Démocratique du Congo : Note de Plaidoyer. Forest People Programme.

18. Lyamahesana, J.-C. K. (2013). Les pygmées riverains des aires protégées : des peuples soumis aux nouvelles formes d'esclavage. Cas du parc national de Kahuzi-Biega en République Démocratique Congo.

19. Matabaro, M. (2009). La crise foncière à l'Est de la RDC. L'Afrique des Grands Lacs.

20. Matarrita-Cascante, D., Trejos, B., Qin, H., Joo, D., \& Debner, S. (2017). Conceptualizing community resilience : Revisiting conceptual distinctions. Community Development, 48(1), 105-123.

21. MECNT, M. de l'Environnement et C. de la N. et tourisme. (1997). Premier rapport national sur la mise en cuvre de la CDB en République Démocratique $d u$ Congo, Kinshasa. (http://www.cbd.int/doc/world/cd/cd-nr-cpb-01-fr.pdf).

22. MECNT, M. de l'Environnement et C. de la N. et tourisme. (2001). Deuxième rapport national sur la mise en cuvre de la CDB en République Démocratique $d u$ Congo, Kinshasa (http://www.cbd.int/doc/world/cd/cd-nr-02-fr.pdf).

23. MECNT, M. de l'Environnement et C. de la N. et tourisme. (2005). Troisième rapport national sur la mise en ceuvre de la $C D B$ en République Démocratique $d u \quad$ Congo (http://www.cbd.int/doc/world/cd/cd-nr-03-en.pdf). 
24. MECNT, M. de l'Environnement et C. de la N. et tourisme. (2009). Quatrième rapport national sur la mise en ouvre de la $C D B$ en République Démocratique du Congo.

25. MECNT, M. de l'Environnement et C. de la N. et tourisme. (2014). Cinquième rapport national sur la mise en cuvre de la $C D B$ en République Démocratique $d u \quad$ Congo. (https://www.cbd.int/doc/world/cd/cd-nr-05-fr.pdf).

26. Nelson, J., \& Hossack, L. (2003). From principles to practice: Indigenous peoples and protected areas in Africa. Morteon-in Marsh, UK: Forest Peoples Programme.

27. Ngono, J. P. N. (2019). Résilience des Baka du Cameroun: Un défi contemporain.

28. Norris, F. H., \& Stevens, S. P. (2007). Community resilience and the principles of mass trauma intervention. Psychiatry: Interpersonal and Biological Processes, 70(4), 320-328.

29. Sandlos, J. (2008). Not wanted in the boundary: The expulsion of the Keeseekoowenin Ojibway band from Riding Mountain National Park. Canadian Historical Review, 89(2), 189-221.

30. Sylvain, L. (2002). Le Guide d'entrevue son élaboration, son évolution et les conditions de réalisation d'une entrevue. 023147 Actes du 12e Colloque de l'ARC.

31. Tauli-Corpuz, V. (2016). Report of the Special Rapporteur of the Human Rights Council on the rights of indigenous peoples. Available at: unsr. vtaulicorpuz. org/site/index. php/en/documents/annualreports/149-report-ga-2016.

32. Wilson, G. (2012). Community resilience and environmental transitions. Routledge.

33. Zautra, A., Hall, J., \& Murray, K. (2008). Community development and community resilience: An integrative approach. Community Development, 39(3), 130-147. 